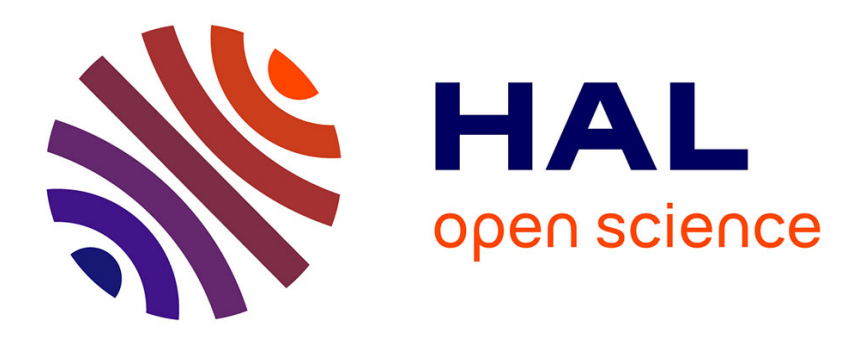

\title{
Adaptive virulence evolution: the good old fitness-based approach
}

\author{
Samuel Alizon, Yannis Michalakis
}

\section{To cite this version:}

Samuel Alizon, Yannis Michalakis. Adaptive virulence evolution: the good old fitness-based approach. Trends in Ecology and Evolution, 2015, 30 (5), pp.248-254. 10.1016/j.tree.2015.02.009 . hal-01567907

\section{HAL Id: hal-01567907 \\ https://hal.science/hal-01567907}

Submitted on 5 May 2018

HAL is a multi-disciplinary open access archive for the deposit and dissemination of scientific research documents, whether they are published or not. The documents may come from teaching and research institutions in France or abroad, or from public or private research centers.
L'archive ouverte pluridisciplinaire HAL, est destinée au dépôt et à la diffusion de documents scientifiques de niveau recherche, publiés ou non, émanant des établissements d'enseignement et de recherche français ou étrangers, des laboratoires publics ou privés. 


\title{
Adaptive virulence evolution: the good old fitness-based approach
}

\author{
Samuel Alizon and Yannis Michalakis \\ Laboratoire MIVEGEC (UMR CNRS 5290, IRD 224, UM1, UM2) \\ 911 avenue Agropolis, B.P. 64501, 34394 Montpellier Cedex 5, France \\ email: samuel.alizon@cnrs.fr yannis.michalakis@ird.fr Phone: +33.4.67.41.64.36
}

Keywords: virulence, evolution, trade-off, epidemiology, fitness, pathogen

\section{Highlights:}

- Parasite virulence can be adaptive

- An approach based on parasite fitness allows to directly test this

- This avoids caveats of tests of the transmission-virulence trade-off hypothesis

\begin{abstract}
Infectious diseases could be expected to evolve towards complete avirulence to their hosts if given enough time. This is not the case. Quite often, virulence is maintained because it is linked to adaptive advantages to the parasite, which is often associated with the hypothesis known as the transmission-virulence trade-off. We argue that this hypothesis currently suffers from three limitations, which have to do with the way virulence is defined, the possibility for multiple trade-offs and the difficulty to test it empirically. Adopting a fitness based approach, where the relationship between virulence and the fitness of the parasite throughout its life cycle is directly assessed, addresses these limitations and allows answering directly whether virulence is adaptive or not.
\end{abstract}

(117 words) 


\section{Why do infectious diseases harm their hosts?}

The maintenance of parasite virulence, which is most generally defined as a decrease in host fitness due to the infection, is a longstanding puzzle [1-9]. Even if there are exceptions known as obligate killing parasites, host death appears as an a priori costly event for parasites as it also results in their own death and thus the end of the infectious period and transmission.

One theoretical possibility to explain that a parasite population does not eventually evolve towards complete avirulence is that the trait cannot evolve due to the lack of relevant genetic variation. Virulence can also persist if it is neutral (i.e. neither selected for or against). This could occur for example if the host is a 'dead-end' to the parasite, in which case any harm to the host does not affect parasite spread. The final possibility, on which we focus here, is that virulence is maintained through natural selection because this trait directly or indirectly confers an advantage to the parasite strain expressing it.

The most common hypothesis to explain the adaptive scenario is the transmission-virulence trade-off hypothesis, which states that strains with increased transmission ability cause shorter infections. This hypothesis has been so successful that most empirical tests of whether virulence can be adaptive for the parasite have focused on testing the existence of a positive relationship between proxies for virulence and transmission [10-16].

We argue here that the way the transmission-virulence trade-off hypothesis is currently formulated entails three major problems when attempting to prove that virulence is adaptive. First, the hypothesis relies very strongly on the way transmission and virulence are defined. Second, it neglects 'hidden' trade-offs and thus paves the way to pan-adaptationist interpretations. Third, testing the existence of the relationship between virulence and transmission is complicated. Here, we propose to recast the problem in its original formulation: looking at the relationship between virulence and parasite fitness, as a few experimental studies have already done and as we propose here, curtails the limitations of the transmission-virulence trade-off hypothesis and answers directly the question of whether virulence is adaptive or not. Adequately defining parasite fitness requires addressing all the relevant life-history traits of a parasite, and mathematical modeling can greatly help in that stage.

\section{Adaptive bases of virulence}

\section{The transmission-virulence trade-off}

In 1943, Gordon Ball [2] challenged the avirulence theory, which states that given enough time parasites should coevolve with their hosts to become avirulent. His opinion was based on evidence that some parasites have maintained their virulence over long periods of time, but he lacked an alternative theory. The latter emerged in the early 80s when Ewald [4] and Anderson and May [3] argued that virulence can be adaptive if its value correlates with other infection traits that are beneficial to parasite transmission. implicit idea is that in order to be transmitted, parasites need to exploit (and hence harm) their host. This is now known as the transmission-virulence trade-off hypothesis.

The fact that the term 'trade-off' is used to describe a positive correlation between virulence and transmission is often confusing to evolutionary biologists, a trade-off between two traits 
intuitively implying a negative relationship. The use of the term probably comes from the fact that virulence is inversely proportional to the duration of the infection, and that an intermediate optimum arises only if at some point the benefits (increased transmission rate) increase less rapidly than the costs (increase host mortality; [17]).

Several studies have provided empirical support to the transmission-virulence relationship (reviewed in [18]). Among these, three host-parasite associations stand out: myxoma virus in rabbits [3,10], HIV in humans [13] and Cauliflower mosaic virus in Brassica rapa [15]. Not only do these show a positive correlation between virulence and transmission but they also find that there exists an intermediate optimal level of virulence. Indeed, they find that the transmission-virulence trade-off curve saturates, which implies that at some point the benefits, that is increased transmission, increase more slowly than the costs (Figure 1A).

\section{Other explanations}

Another adaptive explanation that accounts for the evolution of parasite virulence involves multiple infections (reviewed in [19]). It states that if more virulent strains are more competitive within a host, then allowing for parasites to infect already-infected hosts can maintain virulence, even in absence of a transmission-virulence trade-off [20].

Increased virulence could also occur if parasites can evolve over the course of an infection and if more virulent strains are better adapted to the within-host level. This process has been termed 'short-sighted' evolution [21] because virulence can evolve even if it does not provide any benefit for transmission (or, more generally, any benefit at the between-host level).

Finally, if one allows for more complicated parasite life cycles, several other sources for the maintenance of parasite virulence emerge. For instance, if parasites have a free-living state, there can be coincidental selection and virulence in the focal host can be maintained if it provides an advantage to the parasite in the environment $[22,23]$.

\section{Three limitations of the current trade-off theory}

\section{Virulence and transmission definitions are very stringent}

The initial formalization that led to the transmission-virulence trade-off hypothesis, and the great majority of subsequent formalizations, considered horizontally and directly transmitted parasites $[3,24]$. As a consequence, by 'transmission' the theory means transmission rate (that is the product between the contact rate between hosts and the transmission probability per contact) and by 'virulence' it means the increase in host mortality rate due to the infection. Indeed, in horizontally transmitted parasites, infection effects on host reproduction do not affect parasite fitness, and thus should not influence virulence evolution unless the contacts in the host populations are structured $[25,26]$.

The fact that virulence is expressed as the host mortality rate in the theoretical models on which the transmission-virulence hypothesis was built on is not a secondary issue as measuring virulence experimentally through host case mortality, lethal dose or host life expectancy (which are all related to host mortality) can yield different results. For instance, if the host background mortality rate increases, parasite-induced mortality rate, i.e. the 'virulence' of the 
mathematical models, is expected to increase though, perhaps counter-intuitively, case mortality might decrease [27]. This result makes it easy to envision how sub-lethal measures of virulence (e.g. anemia or weight loss), which are often used for practical reasons, could yield very different predictions than those produced by the measure of virulence used in the theoretical models underlying the trade-off hypothesis.

\section{There can be multiple trade-offs}

Even for parasites that follow the simple life cycle assumed by the models underlying the transmission-virulence trade-off hypothesis (with horizontal and direct transmission), there might be 'hidden' trade-offs. For instance, one might conclude from the absence of a relationship

between the probability of transmission and the parasite-induced mortality rate that there is no trade-off. However, it could be that the cost of an increased probability of transmission is paid through a decrease in contact rate (a hypothesis put forward originally by Ewald [4]).

The problem becomes even more acute for parasites with life cycles that depart from the classical simple one. Let us consider spore-transmitted parasites for instance. Their life cycle can be described as follows: infected hosts produce spores, these spores need to survive long enough to encounter a susceptible host and infect this host upon contact. We could of course consider the trade-off between the probability of infection and virulence but by doing so we could be ignoring a great deal of the biology. What if the trade-off involves virulence and spore production rate or spore survival rate instead?

This multiplicity of trade-offs raises two problems. First, even if we do find a trade-off between two variables of the life cycle, how can we be sure that this is not going to be canceled by another trade-off that involves other variables? For instance, if we find that more virulent spores cause infections that produce less spores, we might conclude that virulence is not adaptive. But what if the quality of the spores (how long they persist in the environment) increases with virulence, as supported by data on human respiratory pathogens [28]? Then, perhaps a more virulent strain that produces less spores but of extremely high quality would be fitter than a less virulent strain. The inadequacy of studying trade-offs between specific fitness components rather than 'total fitness' has already been highlighted in other fields of evolutionary biology [29].

The converse problem occurs if one does not find a trade-off between two variables because proponents of the trade-off hypothesis could be tempted to argue that this is because not all the possible trade-offs have been investigated. Such a pan-adaptationist argument would make the trade-off hypothesis virtually untestable for many parasites with a complicated life-cycle.

\section{Measuring the trade-off is complicated}

A final issue with the current theory occurs when trying to assess the shape of the transmissionvirulence trade-off curve (if any) for a given host-parasite interaction. Importantly, the trade-off curve arises from within-host interactions. Although variations in epidemiological factors such as the number of susceptible hosts have no effect on the trade-off shape, host differences do. In order to minimise these variations, it is highly desirable to study parasites while minimizing environmental variation and variation due to differences due to the hosts, be they genetic or environmental [30]. 
Then, one needs parasite strains that differ in their virulence. These should be sufficiently related because the implicit assumption is that they compete for the same niche and can replace one another. A potential problem is that different isolates might have different evolutionary histories and might thus be more or less (mal)adapted to exploiting the host in the environmental conditions set by the experiment. For example, assume a researcher is using a set of parasitic isolates collected in different parts of the distribution of the species under study. Assuming that the transmission-virulence trade-off hypothesis holds for this species, some of these isolates might be well adapted to the experimental conditions and the variation they will exhibit will lie around the transmission-virulence curve that the experiment aims at inferring. However, other isolates might be particularly maladapted, and appear as outliers of the curve that we try to infer.

\section{A fitness-based approach}

We argue that adopting an approach based on the fitness of the parasite can address the limitations listed above. By doing so, we go back to the roots of the transmission-virulence trade-off hypothesis, which stems from an epidemiological model (p. 499 in [24]).

Obviously this approach requires a definition of parasite fitness (see Box 1 for a formal definition) and ways to measure it (see Box 2). One of the key features of this approach is that the entire life cycle of the parasite is closed and explicitly considered. Therefore, by definition, we are sure that a trait value (usually the virulence) that maximises fitness is evolutionarily stable.

The fitness-based approach has already been applied to empirical data in a very small number of cases. HIV provides a striking example where intermediate virulence values maximise parasite fitness [13]. Arguably, HIV offers a simple setting since parasite fitness has two components, the duration of the infection and the transmission rate. However, several examples from the literature lead us to think that this approach can be widely applicable to biological systems.

For instance, Chapuis et al. [31] showed that intermediate virulence maximises parasite fitness in the case of a bacterium that can alternate between two hosts (an insect and a nematode vector). Another example is provided by Mackinnon and Read [11], who expressed the fitness of Plasmodium falciparum infections by analysing several components separately. In the latter case, they found that the composition of the host population (the proportion of children) strongly affects parasite fitness.

But the best example of the wide applicability of the fitness-based framework is probably the study by de Roode et al. [32]. In their system, the parasite (the protozoan Ophryocystis elektroscirrha) infects monarch butterflies (Danaus plexippus). Typically, adults do not die from the disease but the infection is still virulent because it can decrease the probability of successful emergence from pupal cases. The transmission of the parasite mainly occurs when infected females lay eggs. Parasite spores are then found on the egg and the leaf and larvae become infected by ingesting these spores. In this system, directly measuring the number of secondary infections is complicated so the authors define fitness as the product between five 'components': the probability of adult emergence of an infected larva, the probability of mating, the proportion of eggs that acquire parasite spores and the proportion of offspring that will become infected. 
By doing so, they close the life-cycle of the parasite and thus measure the number of secondary infections. Overall, they show that intermediate spore loads (their proxy for virulence) can maximise parasite fitness (Figure 1B).

\section{The fitness-based framework}

\section{The idea}

For any parasite, it is possible to investigate the relationship between the harm it does to its host and the efficiency with which it spreads. Under this approach, the first quantity, i.e. the parasite's virulence, can be measured in any way that is relevant to the biology of the hostparasite interaction. The second quantity is the parasite's fitness (Box 1). This approach is sufficient to show whether a given level of virulence is maintained by natural selection, which would be the conclusion if fitness is maximised for non-minimal levels of virulence. It would open the way to investigations on the various mechanisms throughout the host and the parasite's life cycles responsible for the benefits virulence confers to the parasite.

We argue that looking at the correlation between parasite fitness and virulence instead of the correlation between transmission rate and virulence addresses all the three major limitations currently faced by the virulence evolution theory.

\section{Wide applicability}

The current transmission-virulence trade-off theory can (and should) only be used for parasites that are transmitted directly. This is frustrating because if virulence can be adaptive for such parasites, it seems logical to assume that it could also be adaptive for parasites transmitted by spores or by vectors. However, a direct translation of the theory to parasites with different life cycles can lead to erroneous results. It is necessary to 'patch' the 'standard' model such that the predictions tested are generated by models that adequately take into account the biology of the systems under study. This may sound like a trivial remark, but unfortunately it often happens that the predictions of the model assuming horizontally transmitted parasites with a direct life cycle are applied to systems with very different biology. Models addressing various deviations from the 'default' horizontally and directly transmitted parasites exist (e.g. [24]) but have rarely been used to make predictions on the evolution of virulence. The advantage of the fitness-based approach is that it relies on a quantity, fitness (Box 1), that can be measured for any parasite with a known life cycle (Box 2), and will yield reliable results provided the entire life cycle is studied, i.e. fitness, and not simply a component of, is measured. In fact, even if part of the life cycle of the parasite is unknown, as is the case for many opportunistic pathogens that have a free-living stage [23], a fitness expression can still be written, even if it might lack in accuracy. Interestingly, the fitness-based approach encompasses almost all the adaptive interpretations of virulence mentioned earlier because as long as parasite fitness can be measured, it is possible to study its relationship to virulence.

Another situation that fits into the framework in a way that could be seen as less intuitive is multiple infections. As shown in previous studies, parasite fitness can be written for superinfection models and for most coinfection models [17,20,33]. An intuitive interpretation of the 
underlying trade-offs is that virulence correlates with within-host competitiveness. Importantly, if multiple infections are allowed, the fitness of a strain de facto becomes a relative measure as it depends on the nature of the resident strain already present in the system. One possibility to circumvent this problem is to use experimental evolution and compare scenarios with or without multiple infections [19].

\section{Get the main picture before the details}

Currently, many studies only investigate the existence of a potential trade-off between virulence and transmission. This approach can lead to false negative results, not only because some level of virulence may be adaptive even if there is no correlation between transmission and virulence but also because the relationship between virulence and transmission can be blurred by correlations with other traits. It can also lead to false positive results, because the effects of a transmissionvirulence trade-off might be cancel out by correlations with other elements of the parasite's fitness.

These effects are difficult to predict. For instance, we mentioned that data on human respiratory pathogens find a negative correlation between virulence and survival time in the environment [28]. However, data on phages suggests a negative correlation between virus persistence in the environment and growth rate in host cells [34,35]. In fact, Goldhill and Turner [36] argue that that this difference between viruses of Eucaryotes and phage might be more general.

The fitness-based approach addresses directly the question of whether virulence is adaptive. Investigating the detailed correlations between various elements of the parasite life cycle is only the second step. This is particularly helpful, at least conceptually, for parasites with complex life cycles, since the multitude of potential trade-offs between all the elements of the parasite's life cycle are merged into a single expression (parasite's fitness).

\section{Solving the virulence definition problem}

Embracing the entire parasite life cycle also addresses the recurrent problem of choosing an appropriate measure of virulence. In evolutionary biology, virulence is a quantitative trait most generally defined as the decrease in host fitness caused by the infection [5]. Since host fitness combines reproduction and survival, there are many ways to measure virulence. The problem is that the formulation of the transmission-virulence trade-off hypothesis is very specific as to how virulence should be measured, and depends on the biology of the system: considering components which are not relevant to parasite fitness, such as e.g. any other measure than the host increase in mortality rate due to the infection for the "canonical" horizontally and directly transmitted parasites, can lead to erroneous interpretations. Furthermore, there is an ongoing debate as to whether sub-lethal measures of virulence such as decline in red-blood cells [37] or castration [12] are adequate proxies of mortality rate to study parasite evolution.

The fitness-based approach solves this problem because we can study the relationship between parasite fitness and any virulence measure (or any relevant trait for that matter). 


\section{Graphical representation}

Arguably, part of the misunderstandings in the field of virulence evolution come from the difficulties to interpret the transmission-virulence trade-off curve. More specifically, showing a positive correlation between transmission and virulence is not sufficient to conclude that selection favours an intermediate level of virulence. Indeed, if the relationship is linear, theory predicts that ever increasing levels of virulence will always be favoured. It is only if the relationship saturates, that is, if at some point the benefits (increased transmission rate) increase less rapidly than the costs (increase host mortality), that there can exist an intermediate optimal level of virulence [17]. Note that transmission rate does not need to decrease with virulence for there to be a finite optimal level of virulence.

The fitness-based approach solves these problems by providing a more intuitive representation of the situation in that it directly shows whether parasite fitness is maximised for an intermediate value of virulence or not. In the case of the study by de Roode et al. [32], a classical trade-off approach would for instance have considered the correlation between the probability of adult emergence (a good proxy for virulence) and the proportion of infected offspring, which seems the closest to a transmission function (note that by doing so one would clearly violate the assumptions of the transmission-virulence trade-off model regarding the underlying model). This is shown in Figure 1A and it is difficult to interpret. Conversely, analysing the parasite fitness as a function of parasite load provides a much more precise idea of the adaptiveness of virulence (Figure 1B).

\section{Conclusion}

Rooting virulence evolution in the ecology and epidemiology of the parasite allows addressing directly and conclusively the adaptive nature of virulence. In passing, it also allows solving many of the ongoing controversies regarding the validity of the trade-off hypothesis. More generally, once the adaptive, or not, nature of virulence is clarified, studying trade-offs is certainly useful

for understanding the mechanisms linking virulence to fitness, and potentially for controlling virulence.

\section{Ackowledgments}

We are very grateful to Jaap de Roode for sharing his data with us. 


\section{References}

[1] T. Smith, Some problems in the life-history of pathogenic microorganism, Science 20 (520) (1904) 817-832.

[2] G. H. Ball, Parasitism and evolution, Am. Nat. 77 (771) (1943) 345-364.

[3] R. M. Anderson, R. M. May, Coevolution of hosts and parasites, Parasitology 85 (1982) 411-426.

[4] P. W. Ewald, Host-parasite relations, vectors, and the evolution of disease severity, Annu. Rev. Ecol. Evol. Syst. 14 (1983) 465-485.

[5] A. Read, The evolution of virulence, Trends Microbiol. 2 (3) (1994) 73-76.

[6] S. A. Frank, Models of parasite virulence, Q. Rev. Biol. 71 (1996) 37-78.

[7] D. Ebert, E. A. Herre, The evolution of parasitic diseases, Parasitol. Today 12 (3) (1996) 96-101.

[8] P. Schmid-Hempel, Evolutionary Parasitology: The Integrated Study of Infections, Immunology, Ecology, and Genetics, Oxford Univ Press, Oxford, UK, 2011.

[9] J. J. Bull, A. S. Lauring, Theory and empiricism in virulence evolution, PLoS Pathog 10 (10) (2014) e1004387.

[10] G. Dwyer, S. A. Levin, L. Buttel, A simulation model of the population dynamics and evolution of myxomatosis, Ecological Monographs 60 (4) (1990) 423-447.

[11] M. J. Mackinnon, A. F. Read, Virulence in malaria: an evolutionary viewpoint, Philos. Trans. R. Soc. B 359 (2004) 965-986.

[12] K. H. Jensen, T. Little, A. Skorping, D. V. Ebert, Empirical support for optimal virulence in a castrating parasite, PLoS. Biol. 4 (7) (2006) e197.

[13] C. Fraser, T. D. Hollingsworth, R. Chapman, F. de Wolf, W. P. Hanage, Variation in HIV-1 set-point viral load: epidemiological analysis and an evolutionary hypothesis, Proc. Natl. Acad. Sci. USA 104 (44) (2007) 17441-17446.

[14] L. Råberg, Infection intensity and infectivity of the tick-borne pathogen Borrelia afzelii, J Evol Biol 25 (7) (2012) 1448-53.

[15] J. Doumayrou, A. Avellan, R. Froissart, Y. Michalakis, An experimental test of the transmission-virulence trade-off hypothesis in a plant virus, Evolution 67 (2) (2013) 477486.

[16] P. D. Williams, A. P. Dobson, K. V. Dhondt, D. M. Hawley, A. A. Dhondt, Evidence of trade-offs shaping virulence evolution in an emerging wildlife pathogen, J Evol Biol 27 (6) (2014) 1271-8. 
[17] M. van Baalen, M. W. Sabelis, The dynamics of multiple infection and the evolution of virulence, Am. Nat. 146 (1995) 881-910.

[18] S. Alizon, A. Hurford, N. Mideo, M. van Baalen, Virulence evolution and the trade-off hypothesis: history, current state of affairs and the future, J. Evol. Biol. 22 (2009) 245-259.

[19] S. Alizon, J. C. de Roode, Y. Michalakis, Multiple infections and the evolution of virulence, Ecol. Lett. 16 (4) (2013) 556-567.

[20] S. Levin, D. Pimentel, Selection of intermediate rates of increase in parasite-host systems, Am. Nat. 117 (1981) 308-315.

[21] B. R. Levin, J. J. Bull, Short-sighted evolution and the virulence of pathogenic microorganisms, Trends Microbiol. 2 (3) (1994) 76-81.

[22] B. R. Levin, C. Svanborg Edén, Selection and evolution of virulence in bacteria: an ecumenical excursion and modest suggestion, Parasitology 100 (1990) S103-15.

[23] S. P. Brown, D. M. Cornforth, N. Mideo, Evolution of virulence in opportunistic pathogens: generalism, plasticity, and control, Trends Microbiol 20 (7) (2012) 336-42.

[24] R. M. Anderson, R. M. May, The population dynamics of microparasites and their invertebrate hosts, Philos Trans R Soc Lond B 291 (1054) (1981) 451-524.

[25] J. O'Keefe, K., J. Antonovics, Playing by different rules: the evolution of virulence in sterilizing pathogens, Am. Nat. 159 (6) (2002) 597-605.

[26] S. Lion, S. Gandon, Evolution of spatially structured host-parasite interactions, J Evol Biol 28 (1) (2015) 10-28.

[27] T. Day, On the evolution of virulence and the relationship between various measures of mortality, Proc. R. Soc. Lond. B 269 (2002) 1317-1323.

[28] B. A. Walther, P. W. Ewald, Pathogen survival in the external environment and the evolution of virulence, Biol. Rev. 79 (04) (2004) 849-869.

[29] J. Hunt, L. F. Bussière, M. D. Jennions, R. Brooks, What is genetic quality?, Trends Ecol Evol 19 (6) (2004) 329-33.

[30] S. Alizon, M. van Baalen, Emergence of a convex trade-off between transmission and virulence, Am. Nat. 165 (6) (2005) E155-E167.

[31] E. Chapuis, A. Arnal, J.-B. Ferdy, Trade-offs shape the evolution of the vector-borne insect pathogen Xenorhabdus nematophila, Proc. R. Soc. Lond. B 279 (1738) (2012) 2672-80.

[32] J. C. de Roode, A. J. Yates, S. Altizer, Virulence-transmission trade-offs and population divergence in virulence in a naturally occurring butterfly parasite, Proc. Natl. Acad. Sci. USA 105 (21) (2008) 7489-7494. 
[33] M. Choisy, J. C. de Roode, Mixed infections and the evolution of virulence: effects of resource competition, parasite plasticity and impaired host immunity, Am. Nat. 175 (5) (2010) E105-E118.

[34] M. De Paepe, F. Taddei, Viruses' life history: towards a mechanistic basis of a trade-off between survival and reproduction among phages, PLoS Biol 4 (7) (2006) e193.

[35] R. H. Heineman, S. P. Brown, Experimental evolution of a bacteriophage virus reveals the trajectory of adaptation across a fecundity/longevity trade-off, PLoS One 7 (10) (2012) e46322.

[36] D. H. Goldhill, P. E. Turner, The evolution of life history trade-offs in viruses, Curr. Opin. Virol. 8 (79-84).

[37] M. J. Mackinnon, A. F. Read, Genetic relationships between parasite virulence and transmission in the rodent malaria Plasmodium chabaudi, Evolution 53 (1999) 689-703.

[38] U. Dieckmann, Adaptive dynamics of pathogen-host interactions, in: U. Dieckmann, J. A. J. Metz, M. W. Sabelis, K. Sigmund (Eds.), Adaptive dynamics of infectious diseases: In pursuit of virulence management, Cambridge University Press, 2002, pp. 39-59.

[39] M. W. Sabelis, J. A. J. Metz, Taking stock: relating theory to experiment, in: U. Dieckmann, J. A. J. Metz, M. W. Sabelis, K. Sigmund (Eds.), Adaptive dynamics of infectious diseases: In pursuit of virulence management, Cambridge University Press, 2002, pp. 379398.

[40] T. D. Hollingsworth, R. M. Anderson, C. Fraser, HIV-1 transmission, by stage of infection, J. Infect. Dis. 198 (5) (2008) 687-693.

[41] S. Otto, T. Day, A biologist's guide to mathematical modeling in ecology and evolution, Princeton University Press, 2007.

[42] O. Diekmann, J. Heesterbeek, Mathematical epidemiology of infectious diseases: model building, analysis, and interpretation, Wiley, New York, 2000.

[43] A. Hurford, D. Cownden, T. Day, Next-generation tools for evolutionary invasion analyses, J. R. Soc. Interface 7 (45) (2010) 561-71.

[44] T. Day, S. Alizon, N. Mideo, Bridging scales in the evolution of infectious disease life histories: theory, Evolution 65 (12) (2011) 3448-61.

[45] N. Mideo, W. A. Nelson, S. E. Reece, A. S. Bell, A. F. Read, T. Day, Bridging scales in the evolution of infectious disease life histories: application, Evolution 65 (11) (2011) 3298-310.

[46] F. Luciani, S. Alizon, The evolutionary dynamics of a rapidly mutating virus within and between hosts: the case of hepatitis C virus, PLoS Comput. Biol. 5 (11) (2009) e1000565.

[47] K. A. Lythgoe, L. Pellis, C. Fraser, Is HIV short-sighted? Insights from a multistrain nested model, Evolution 67 (10) (2013) 2769-82. 
[48] C. Fraser, K. Lythgoe, G. E. Leventhal, G. Shirreff, T. D. Hollingsworth, S. Alizon, S. Bonhoeffer, Virulence and pathogenesis of HIV-1 infection: an evolutionary perspective, Science 343 (6177) (2014) 1243727.

\section{Box 1: What is parasite fitness?}

In the case of parasites, several definitions can be found in the literature. For instance, some will equate parasite fitness with parasite load, others with replication rate, others with virulence.

The measures we listed above are all restricted to the individual host level but here we want to identify the parasite strain that is most capable of invading a host population. Therefore, fitness must be defined at the host population level.

One of the easiest ways to estimate fitness in this situation is to measure the ability of the parasite to spread through the host population. In epidemiology, this is also known as the basic reproduction ratio $\left(R_{0}\right)$ and corresponds to the expected number of secondary infections caused over the whole course of an infection (akin to the number of offspring for free living organisms). Note that this definition implicitly accounts for the whole life-cycle of the parasite.

As all fitness measures, this one has some limitations. For instance, there can be differences between the invasion fitness in a naive host population and in a host population already infected by a resident strain of the parasite [38]. Furthermore, the definition does not account for out of equilibrium dynamics [39]. However, this invasion fitness has the advantage to be applicable to most host-parasite systems and to allow confrontation to experimental data (see Box 2).

\section{Box 2: Measuring parasite fitness}

\section{Empirical approach}

The first way to measure parasite fitness is to 'close' the life-cycle in the laboratory. Practically, one would start from a host infected by a parasite strain and count how many hosts have been infected in the population from this initial host. The fittest strain is the strain that generates the highest number of secondary infections.

This experimental might not be feasible for practical reasons in some host-parasite systems. A second way to estimate parasite fitness then consist in dividing the fitness into 'components' that are easier to measure. Note that writing down the mathematical expression of parasite fitness can be instrumental in defining the components and identifying potential correlations between them. For instance, in the case of HIV it is difficult to count the number of secondary infections for a given host. However, the problem can be overcome by noticing that the fitness is equal to the product between two components, which are the duration of the infection and the transmission rate [13].

The main limitation of dividing fitness into components is that one has to be careful as to when the value of a component is measured. Again in the case of HIV the transmission probability per contact varies widely over the course of an infection [40] so ideally this should be reflected on the 'transmission' component. One possibility for instance is to measure values at several time point during the infection. 


\section{Mathematical approach}

Practically, a parasite's life cycle can be captured by a system of differential equations [41]. One can then calculate parasite fitness by differentiating this system to obtain what is known as the Jacobian matrix [42]. The dominant eigenvalue of this matrix indicates if the parasite can spread or not in the host population (see also [43]). In the classical case assumed implicitly by

the trade-off hypothesis, parasites are only transmitted upon contacts between hosts and the fitness $\mathcal{R}$ is:

$$
\mathcal{R}=\frac{\beta}{\mu+\alpha+\gamma}
$$

where $\beta$ is the transmission rate (which is the product of a contact rate between hosts and a probability of transmission per contact), $\mu$ is the host baseline mortality rate, $\alpha$ is the diseaseinduced mortality (or virulence) and $\gamma$ is the recovery rate. Note that, as explained in Supporting Text S1, $\mathcal{R}$ is often equivalent to the basic reproduction ratio $\mathcal{R}_{0}$ but not always [38].

In addition to providing an intuition regarding the fitness components to measure, the mathematical expression of parasite fitness helps to make predictions regarding virulence evolution. From equation 1, strains with low virulence $(\alpha)$ should be favored because they have a higher fitness, $\mathcal{R}$ (the avirulence theory). The transmission-virulence trade-off hypothesis challenges this by stating that virulence $(\alpha)$ and transmission rate $(\beta)$ are positively linked, such that decreasing one decreases the other. As a result parasites will evolve either to an intermediate level of virulence, if the relationship between the two traits saturates, or to the maximal possible value otherwise.

In the Supporting Text S1, we give further details about how to derive mathematical expressions for parasites with a variety of transmission routes. Ideally these should be derived for each system in order to capture the specificities of this system and guide empirical measurements. 


\section{Box 3: Open problems}

\section{Practical limitations}

It is not always easy or even sometimes possible to study the entire life cycle empirically. For instance for opportunistic parasites, we have little (or no) idea of what their ecology outside the host is [23]. Some parasites have complex life cycles; and some of their hosts cannot be raised in the lab, or experiments on them are not ethically acceptable.

One can go even further by making the point that lab conditions will never re-create real ecological conditions. Think for instance of a spore-producing parasite that would face a tradeoff between virulence and spore longevity (as shown for human respiratory viruses [28]). Even if we manage to close the life cycle in the lab, how can this trade-off be tested unless the experiment runs for a very long time or unless the input of susceptible hosts is varied? Arguably, this is where mathematical modeling can help. We first need to identify which parameters of the life cycle depend on the epidemiological state of the population in order to infer how optimal levels of virulence can vary in response to environmental changes. Of course, this is assuming that the parasite's life cycle recreated in the lab can be compared to that in nature.

\section{Timing problem}

In vector-borne diseases for instance, the probability that a vector becomes infected after biting an infected host can vary with the age of the infection. One possibility is to study the components of parasite fitness over time and average them to perform the analysis. There even exist some more elaborate methods inspired from quantitative genetics that allow incorporating within-host time series into an epidemiological model [44]. By applying such a framework to Plasmodium chabaudi infections in mice, Mideo et al. [45] showed that transmission early in the infection can be traded-off against transmission later in the infection.

\section{Within-host evolution}

Although multiple infections can readily be included in this framework, it is more challenging to include within-host evolution. The main problem with this process is that the parasite strains that come out of the host can strongly differ from those that infected it [46]. However, recent models that include biological features of the parasite seem to offer promising perspectives by assuming an explicit within-host fitness landscape and deriving a next-generation epidemiological model [47]. Also, recent findings suggest that even in rapidly evolving parasites causing persistent infections, virulence could be highly 'heritable' from one infection to the next [48], thus suggesting that within-host evolution could be less of a problem than previously hypothesized $[21]$. 

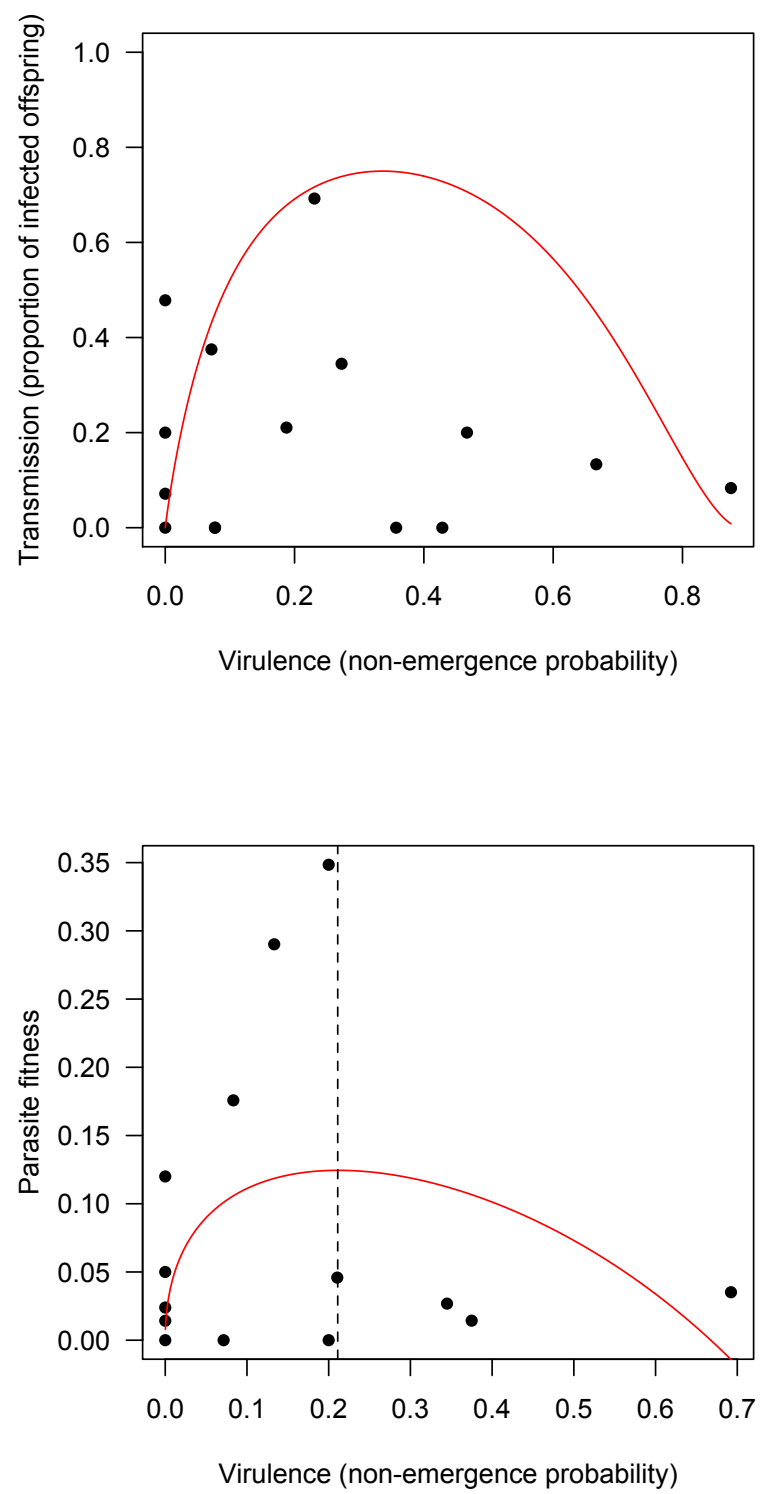

Figure 1: Graphical representation of the trade-off curve (A) and of the parasite fitness (B). Data originates from the work by de Roode et al. [32] on a protozoan parasite infecting monarch butterflies. The lines show the best model that fit the data best based on the Akaike Information Criterion. A) A proxy of transmission as a function of a proxy of virulence. The evolutionarily stable (ES) virulence cannot be inferred directly from this curve because the host baseline mortality is unknown. B) Parasite fitness as estimated by de Roode et al. through the measurement of four fitness components as a function of the same proxy of virulence. The dashed line shows the ES virulence, which by definition maximises parasite fitness. Note that fitness could not be computed for all the isolates. Further details about the figure and this system are available in Supplementary Text S1. 